\title{
La Anatomía Humana como Disciplina Indispensable en la Seguridad de los Pacientes
}

\author{
Human Anatomy an Essential Discipline for Patient Safety \\ Robinson Rodríguez-Herrera ${ }^{1,4,6}$; Ricardo J. Losardo ${ }^{2,5,7}$ \& Octavio Binvignat $^{3}$
}

\begin{abstract}
RODRÍGUEZ-HERRERA, R.; LOSARDO, R. J. \& BINVIGNAT, O. La Anatomía humana como disciplina indispensable en la seguridad de los pacientes. Int. J. Morphol., 37(1):241-250, 2019.
\end{abstract}

RESUMEN: En este artículo se resaltó la importancia que tiene un buen conocimiento de la anatomía humana para una mejor práctica médica, brindando al paciente una mayor seguridad.

PALABRAS CLAVE: Anatomía humana; Seguridad del paciente; Error médico; Educación médica.

“Nulla Medicina Sine Anatomia” (Di Dio, 1998).

\section{INTRODUCCIÓN}

Actualmente, y desde hace algunos años, se considera que la seguridad del paciente es un principio fundamental de la atención sanitaria. Se entiende que hay un cierto grado de riesgo en este proceso. Por supuesto que el médico es una pieza clave en él. Su formación universitaria no es un tema menor. El plan de estudios de esta Carrera habitualmente comienza con Anatomía, una de las asignaturas más extensas de la curricula.

La Anatomía es la base de la medicina, pues la fisiología, la patología, la semiología y la terapéutica requieren de su conocimiento y del correcto uso del lenguaje anatómico. Por otra parte, a pesar de la gran cantidad de avances médicos y del apoyo de la informática, hoy día pueden producirse errores en la práctica médica, originadas a partir de una inadecuada descripción e interpretación de la anatomía, tanto en los diagnósticos como en los tratamientos. Creemos que, sin duda, el correcto aprendizaje de la anatomía está relacionado con la seguridad del paciente (Fig. 1).

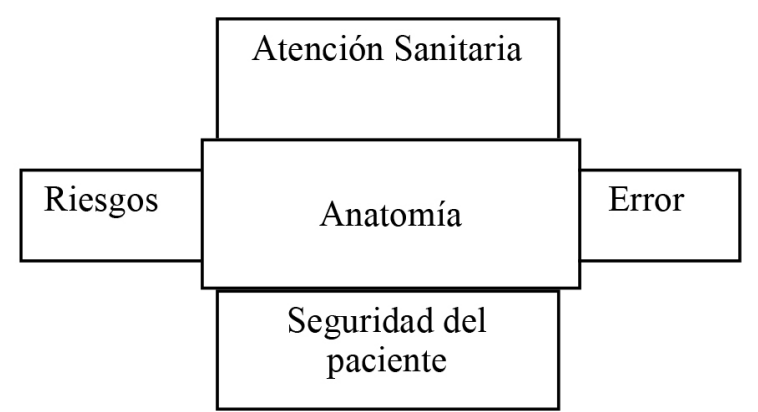

Fig. 1. Anatomía y seguridad del paciente.

\section{LAS ÁREAS DE LA ANATOMÍA}

Algunas divisiones o sub-disciplinas de la anatomía permiten comprender mejor su extensión e importancia; y demuestra que como ciencia básica sigue siendo relevante y constituye un tronco integrador que relaciona distintas temáticas.

- Anatomía Sistémica: inapropiadamente conocida como “Descriptiva”, estudia el cuerpo por sistemas y aparatos.

\footnotetext{
${ }^{1}$ Facultad de Ciencias de la Salud, Universidad Latinoamericana de Ciencia y Tecnología (ULACIT); Facultad de Ciencias de la Salud, Universidad de Iberoamérica (UNIBE). San José, Costa Rica.

${ }^{2}$ Escuela de Posgrado, Facultad de Medicina, Universidad del Salvador (USAL). Buenos Aires, Argentina.

${ }^{3}$ Escuela de Medicina, Universidad de Talca (UT), Chile.

${ }^{4}$ Hospital Nacional de Niños "Carlos Sáenz Herrera". San José, Costa Rica.

${ }^{5}$ Hospital de Oncología "María Curie". Buenos Aires, Argentina.

${ }^{6}$ Programa Institucional de Calidad y Seguridad del Paciente, Caja Costarricense del Seguro Social (CCSS). San José, Costa Rica.

${ }^{7}$ Presidente de la Academia Panamericana de Anatomía; Presidente Honorario de la Asociación Panamericana de Anatomía.
} 
- Anatomía Regional: también llamada “Topográfica”, la estudia por regiones corporales.

- Anatomía Microscópica o Histológica: estudia los tejidos y órganos con la utilización del microscopio. La aparición del microscopio en el siglo XVII permitió descubrir un nuevo mundo anatómico que luego, se extendió a la anatomía patológica.

-Anatomía del Desarrollo: estudia las modificaciones que sufre el organismo desde su origen (concepción) hasta su senectud. La Embriología es una parte de ella y estudia la anatomía del embrión, del feto y del recién nacido (morfogénesis) (ArteagaMartínez \& García-Peláez, 2017).

-Anatomía Funcional: estudia la finalidad de las estructuras. Es por todos conocida la frase que lo explica: "la forma hace la función". La anatomía en relación con las funciones de los órganos y del cuerpo. Forma y función de las estructuras.

-Anatomía de Superficie: es el estudio de las características o morfología superficial del cuerpo (por ejemplo, relieves de huesos y músculos; y proyección de órganos en la piel).

-Anatomía Bioscópica: estudia las estructuras del cuerpo humano a través de instrumentos que se introducen por vías naturales y/o artificiales (endoscopias, laparoscopias, etcétera).

-Neuroanatomía: estudia la anatomía del sistema nervioso.

-Anatomía Clínica: relaciona la anatomía humana con el diagnóstico y el tratamiento de las enfermedades.

-Anatomía Quirúrgica: es el estudio de la anatomía aplicada a la cirugía.

-Anatomía Aplicada: es el estudio de la anatomía aplicada generalmente a la clínica y a la cirugía. También se la denomina "médico-quirúrgico".

-Anatomía Radiológica o Imagenológica: estudia la anatomía del cuerpo y los órganos que la componen mediante radiografías y/ o imágenes. A finales del siglo XIX se descubrieron los rayos $\mathrm{X}$, los que en 1896 dieron origen a la radiografía aplicada a la medicina. La imagenología es un campo más amplio y moderno que incluye otras técnicas y procedimientos además de la radiografía.

-Anatomía Comparada: compara la anatomía humana con la de los animales y permite relacionar la evolución de los seres vivos (filogenia y ontogenia).

-Anatomía Patológica: estudia las enfermedades o patologías de los órganos y sistemas.

-Anatomía Forense: estudia el cuerpo humano en las circunstancias de muerte.

-Anatomía Antropológica o Antropología física o biológica: estudia al ser humano considerando su anatomía biológica, particularidades y evolución.

-Anatomía Artística: estudia la anatomía para uso y fines artísticos.

-Historia de la Anatomía: estudia el avance de los conocimientos anatómicos a través de las épocas, las culturas y los personajes que describieron por primera vez estructuras, órganos, aparatos y/o sistemas.

Como vemos la anatomía es una ciencia básica que abarca distintas ramas o disciplinas con enfoques propios y específicos, que se complementan entre sí. En su conjunto permiten adquirir una visión más global y una comprensión más acabada del estudio del cuerpo humano.
La anatomía proporciona los conceptos necesarios para correlacionar las estructuras con las funciones, normales y anormales; y de esta manera, se vincula con las disciplinas clínicas (Perriard \& Losardo, 2001). La importancia de la Anatomía se sintetiza en la histórica frase: "La Anatomía es a la Fisiología, lo que la Geografía es a la Historia" (Fernelii, 1610).

\section{HISTORIA Y EVOLUCIÓN DE LA ANATOMÍA}

El término "Anatomía" se acuñó en la Edad Antigua y proviene del griego: "ana", hacia arriba, sobre, repetición; "temnein", corte; y está ligado al término latino "disección": cortar y separar (Gardner et al., 1967; Mandarim-de-Lacerda, 2010).

La anatomía fue el inicio de las ciencias biológicas y por supuesto, de las ciencias médicas. La aparición de la anatomía como un "esbozo" de ciencia y arte, surgió en la Prehistoria y en la Edad Antigua, con la observación de restos cadavéricos y con los primeros exámenes de órganos de las víctimas de accidentes (heridas) y de sacrificios, animales y humanos. Respondió a una curiosidad natural del hombre y a una necesidad por el conocimiento del cuerpo humano (Losardo et al., 2016; Moscol-González, 2018).

Se desarrolló en las primeras civilizaciones, con los hindúes (Ayurveda), egipcios (papiro de Ebers), griegos ("Corpus hippocraticum" o "Tratados hipocráticos") y romanos (Galeno de Pérgamo, Asia Menor) y también con los aborígenes de América. Luego en la Edad Media con los árabes ("Canon o Principios de la Medicina" de Avicena); y en la Edad Moderna, nuevamente con los romanos ("De Humani Corporis Fabrica" de Vesalio) (Figs. $2,3,4)$. Debemos destacar que -en el mundo occidentalel movimiento cultural llamado "Humanismo" y "Renacimiento" actuó como bisagra entre las Edades Media y Moderna. Además, con la creación de la imprenta, en 1450, y su popularización, en el siglo XVI, se facilitó la divulgación de las ideas a través de las publicaciones con textos e ilustraciones. La medicina se benefició con ello. Por su parte, la anatomía avanzó mucho en los tres siglos que abarcó la Edad Moderna (siglos XV-XVIII) (Mandarimde-Lacerda; Losardo et al., 2015a).

En América Latina, la enseñanza formal de la Anatomía se inició, en 1634, en la Universidad Mayor de San Marcos de Lima-Perú, que fue la primera universidad americana, fundada en 1551. A mediados del siglo XVII, se crearon otras universidades que enseñaron Medicina, como las de Nueva Granada, Guatemala y Quito. Todas 


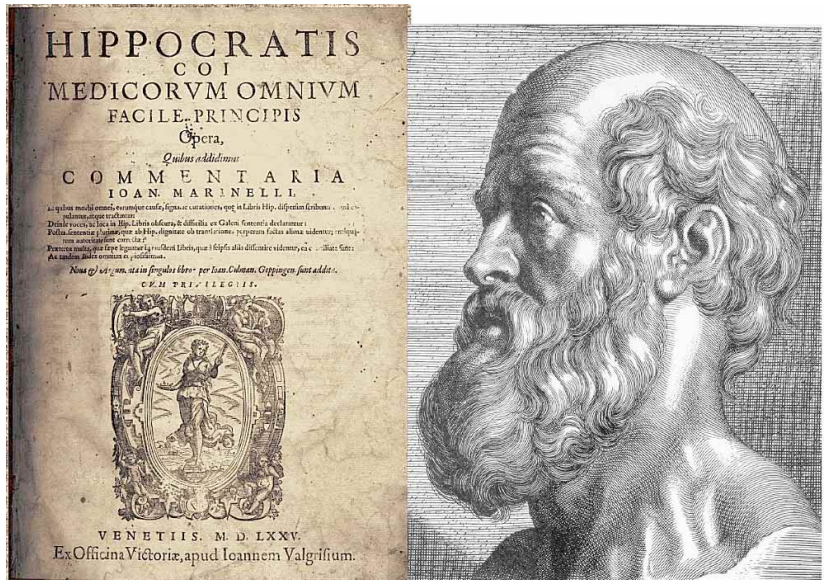

Fig. 2. Corpus hippocraticum e Hipócrates (c.460 aC- c.370 aC)
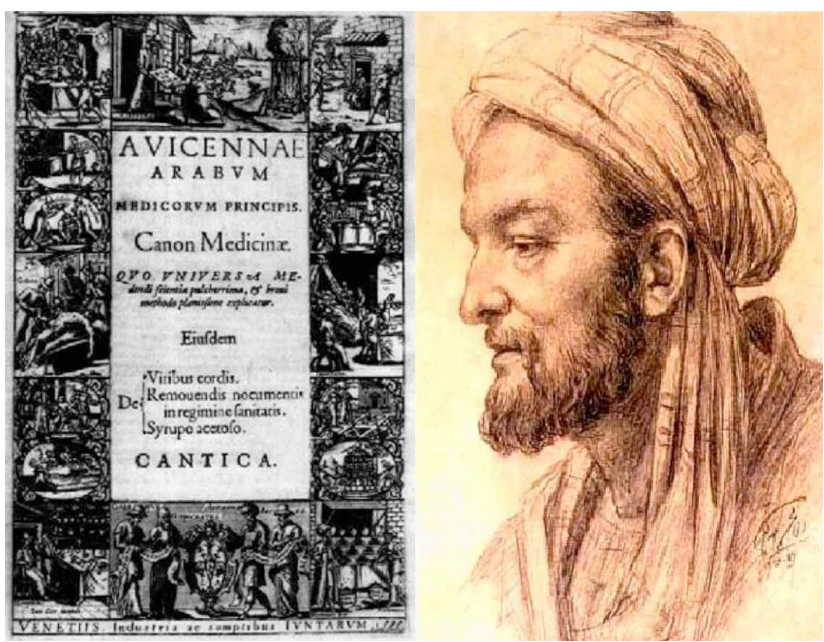

Fig. 3. Canon de la medicina y Avicena (c. 980-1037).
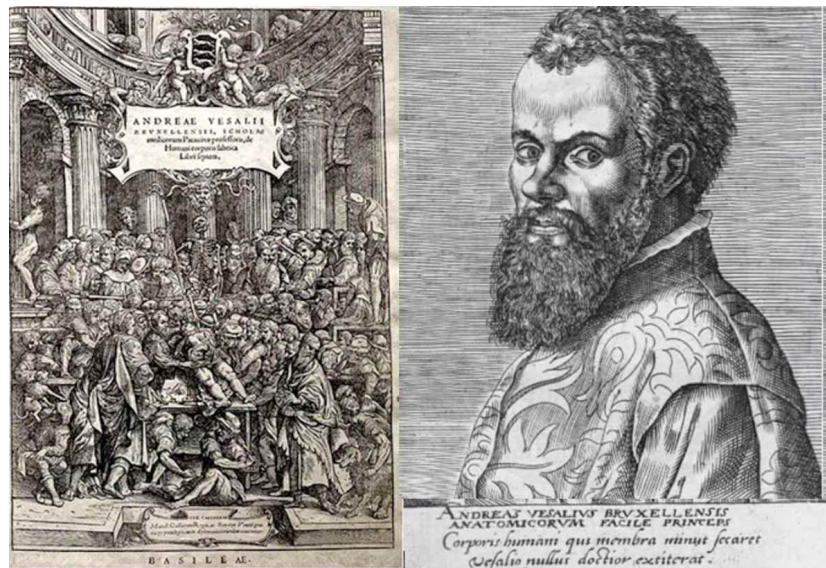

Fig. 4. De Humani Corporis Fabrica y Andrés Vesalio (1514-1564).

ellas con una gran influencia española. Con la independencia de las colonias de España llegaron médicos ingleses, franceses y alemanes que sumaron su experiencia educativa (Bogado-Yinde et al., 2015).
En la Edad Contemporánea, en Europa y América, en el siglo XIX, el estudio de la anatomía macroscópica humana fue culminante y realizó su sistematización, gracias al auge de las investigaciones con las disecciones cadavéricas. Así la anatomía estableció conexiones con la embriología y la histología, interpretándose de una manera más integral.
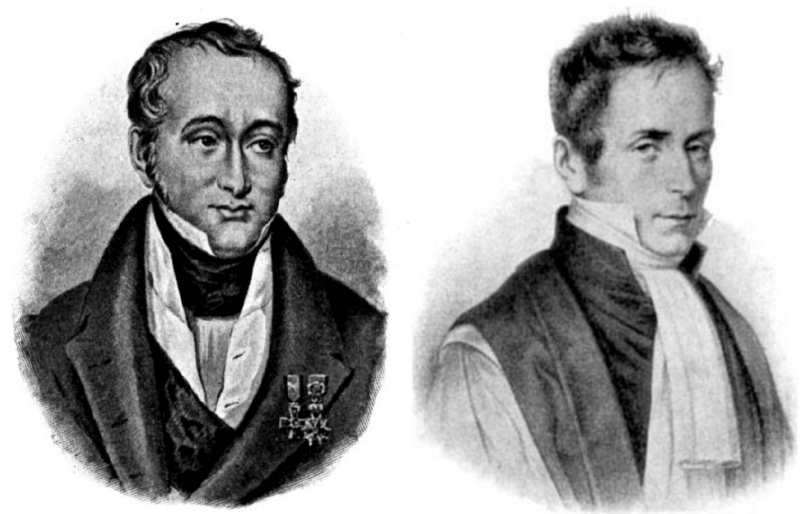

Fig. 5. Guillaume Dupuytren (1777-1835) y René Laennec (17811826).

Para comprender la importancia que tuvo en ese siglo, recordemos que, en 1803, se creó la Sociedad Anatómica de París (Société Anatomique de Paris), de la mano de Guillaume Dupuytren y René Laennec, apoyada por el emperador Napoleón Bonaparte (Fig. 5). Actualmente sigue funcionando en la Universidad René Descartes, en París y es una de las sociedades médicas más antiguas del mundo. Se reconoce que la Anatomía tuvo su máximo desarrollo en el siglo XIX e inicios del siglo XX (Mandarimde-Lacerda). Desde entonces, sigue creciendo con el desarrollo de nuevas tecnologías y el aporte de nuevas investigaciones.

Por otra parte, antes del siglo XX, se estimaba que, en las Escuelas de Medicina del mundo, se utilizaban unos 50.000 nombres para referirse al cuerpo humano y sus componentes, lo que hacía particularmente difícil el aprendizaje, y muy confusa la comunicación entre profesionales, con el inevitable riesgo para la seguridad de aquellos pacientes. Ante tal panorama, en 1895 se convocó a los expertos anatomistas a la ciudad de Basilea, donde se originó el primer intento de unificar términos similares. Este intento fue conocido como la "Nómina Anatómica de Basilea" (NAB). Luego vinieron otros; y en 1950, en la ciudad de Oxford, Inglaterra, se estableció el Primer Comité de la "Nomenclatura Anatómica Internacional". En 1955, durante el Sexto Congreso Mundial de Anatomía, en París, se consolidó el Comité de Nomenclatura Internacional. Y en 1998, tomó forma la "Terminología Anatómica Internacional", y se publicaron los términos de con- 


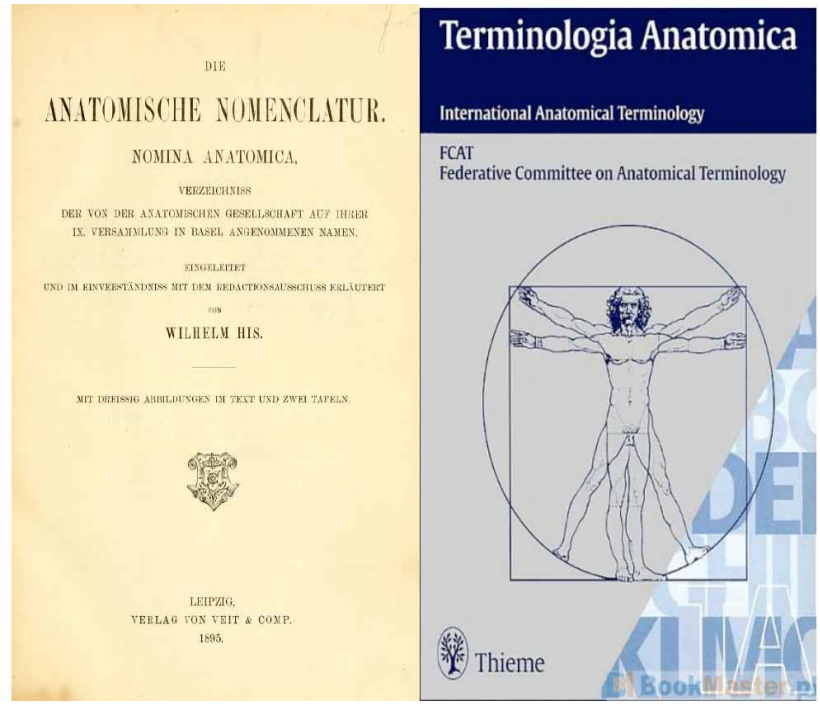

Fig. 6. Nómina Anatómica de Basilea (1895) y Terminología Anatómica Internacional (1998).

senso, que redujeron a la cantidad de 7500 estructuras los elementos macroscópicos del cuerpo. De esta manera, se pudo ordenar y facilitar la comunicación de los conocimientos entre los profesionales (Losardo et al., 2015b) (Fig. 6).

En cuanto a la enseñanza de la anatomía, el espacio que ocupó en el currículo de la Carrera de Medicina a lo largo de la historia fue disminuyendo con el tiempo y fue cediendo lugar al resto de las disciplinas que se desarrollaron posteriormente. El último gran cambio se dio hace más de 60 años, cuando se fusionó la enseñanza de las asignatu-

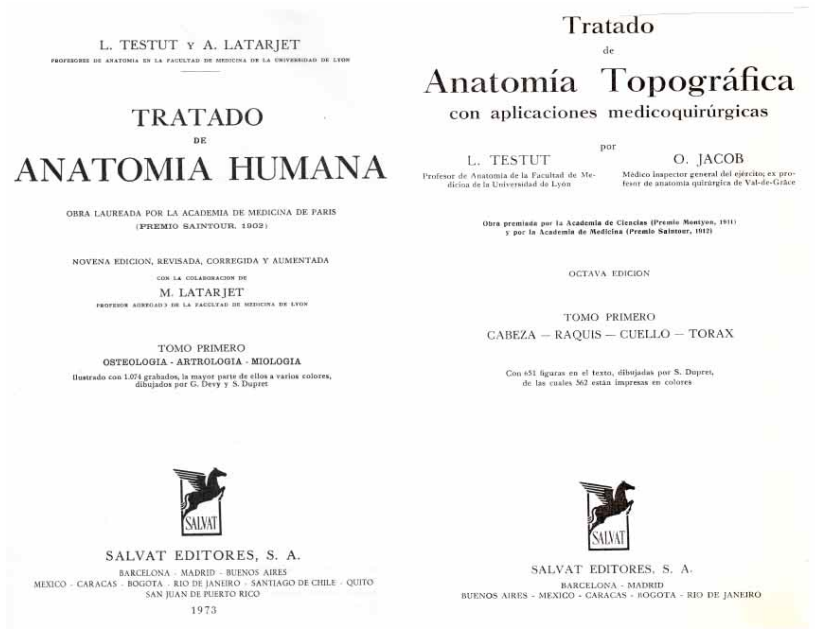

Fig. 7.Libros que se utilizaron para el estudio de la anatomía descriptiva y topográfica durante la primera mitad del siglo XX en la Carrera de Medicina: Tratado de Anatomía Humana (4 tomos) y Tratado de Anatomía Topográfica (2 tomos). Ambos escritos por Léo Testut (1849-1925) en 1892 y en 1921, respectivamente. ras "Anatomía Descriptiva” y "Anatomía Topográfica”. Ambas materias eran anuales y se desarrollaban en el primero y segundo año, respectivamente, permitiendo un acabado conocimiento anatómico, necesarios para el examen físico del paciente y otras prácticas médicas, como las cirugías y los procedimientos mini-invasivos (por ejemplo: tóraco y laparoscopias, endoscopias, artroscopias, cateterismos y punciones) (Fig. 7).

Sin embargo, a medida que, la tecnología médica se fue desarrollando, se incrementó la necesidad de aquellos conocimientos, característica indispensable para lograr una mayor seguridad en la atención del paciente (RodríguezHerrera, 2017a).

\section{LA ENSEÑANZA DE LAANATOMÍA. FORTALEZAS Y DEBILIDADES}

La anatomía como estudio científico del ser humano es una materia compleja y extensa. La anatomía es una ciencia, empírica, positiva y objetiva, por lo tanto, científica. $\mathrm{Su}$ aprendizaje requiere de una dedicación y estudio muy particular; y es indispensable para el médico.

No es sencillo para el joven estudiante, identificar y describir las estructuras, así como comprender y explicar los conceptos morfológicos. Además, los estudiantes necesitan desarrollar un lenguaje descriptivo específico, preciso, universal, inequívoco, denominado Terminología Anatómica (TA). Es un lenguaje utilizado como una herramienta que permite la comunicación entre los profesionales del área de la salud a nivel básico y clínico (Montemayor Flores et al., 2016). Se debe sumar a ella el dominio de la terminología relativa a la planimetría y el espacio tridimensional, los movimientos y la topografía clínica elemental. Finalmente, con "Anatomía" los estudiantes inician el desarrollo de las habilidades y destrezas manuales (competencias) que continuarán a lo largo de su Carrera. Pero a estas áreas cognitiva y psicomotriz del proceso de enseñanza-aprendizaje debemos agregar el área afectiva, que tendrá un valor único en la futura relación médicopaciente (Perriard \& Losardo, 1996). Todo este proceso de aprendizaje demanda concentración y análisis por parte del estudiante, y eso va de la mano del tiempo necesario para incorporar lo aprendido.

La dificultad de estudio que presenta la anatomía como asignatura para los alumnos, se debe fundamentalmente a su extensión y complejidad. Los estudiantes de medicina reclaman más cantidad de días y horas por semana para la cursada de Anatomía, especialmente en las actividades prácticas. Los docentes de experiencia concuerdan al respecto (Farache et al., 1990a). 
La anatomía requiere lectura atenta, observación, examen minucioso de las estructuras, y el uso del léxico correcto en el quehacer diario; primero del estudiante, y luego del graduado. El tiempo y la calidad en el área de disección y museo, revisando las disecciones y modelos anatómicos, utilizando otros instrumentos de investigación tales como el microscopio, moldean la neuro-plasticidad del estudiante, de forma tal, que pueda integrar todo el conocimiento y aplicarlo posteriormente a las etapas siguientes del aprendizaje. Los programas computacionales y otras modalidades de apoyo informático colaboran, pero de ninguna manera sustituyen la lectura detallada y el entrenamiento en el anfiteatro o en el laboratorio, manipulando los cuerpos o las piezas según la guía de los docentes.

Actualmente el perfil del egresado de la Carrera de Medicina ha cambiado. Casi todas las asignaturas han reducido sus contenidos y la formación brindada es inferior a la de otras épocas. En cuanto a anatomía hay una tendencia en algunos de los programas curriculares de enseñarla sólo durante un semestre en el primer año. Este riesgoso desafío se realiza con estudiantes que tienen entre 18 y 19 años, edad por cierto muy temprana para comprender la importancia de su estudio.

Las falencias en los sistemas educativos preuniversitarios y en algunos planes de estudio universitarios conspiran contra el tiempo tan limitado que se dispone, en un semestre, para brindar un buen curso de anatomía general. Además, el docente de anatomía requiere que el estudiante esté adecuadamente capacitado en otras materias básicas, tales como biología, biofísica, bioquímica e investigación, para aprovechar adecuadamente su tiempo de aprendizaje (Farache et al., 1990b). También debe tenerse en cuenta que anatomía no es la única asignatura que matricula en ese período; y por lo tanto, el esfuerzo de aprendizaje es máximo para el estudiante.

Este panorama puede repercutir en la formación y, por consiguiente, en el futuro ejercicio profesional; y vul-

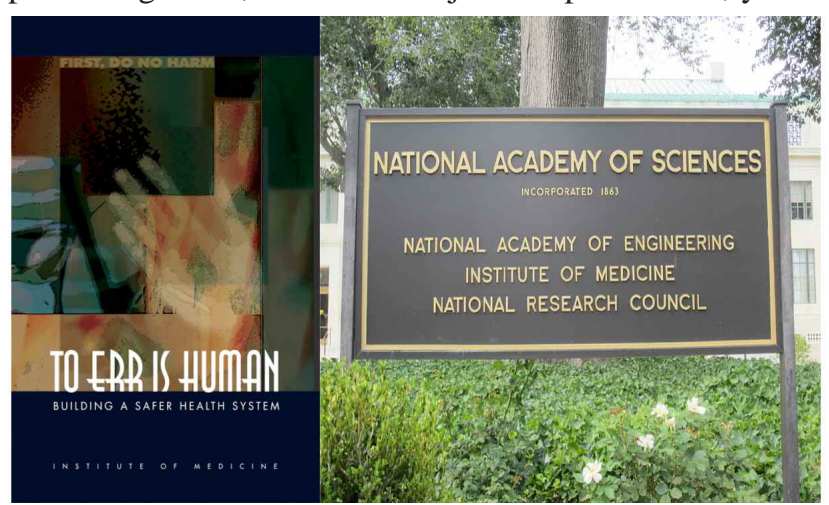

Fig. 8. Informe realizado por el Instituto de Medicina de E.U.A. nerar la calidad y la seguridad de la atención del paciente (Rodríguez-Herrera, 2017a).

\section{LA SEGURIDAD DE LOS PACIENTES Y LA OMS- OPS}

La seguridad del paciente es un proceso dinámico y permanente de gestión en la atención de la salud, destinado a la prevención de riesgos, de forma que los incidentes o eventos adversos sean identificados y documentados científicamente para poder erradicarlos, o disminuir al máximo su eventual aparición e impacto. Todo esto para establecer una atención orientada hacia la mejor calidad en la atención.

En 1999, el Instituto de Medicina de la Academia Nacional de Ciencias de Estados Unidos de Norteamérica (Institute of Medicine, National Academy of Sciencies - IoM, NAS), publicó el informe "Errar es humano: la construcción de un sistema de salud más seguro" (To err is human: building a safer health system); y a partir de él se generó el concepto de "la seguridad de los pacientes" como un principio fundamental en el proceso de la atención sanitaria (Fig. 8).

En el 2002, en Ginebra, la Organización Mundial de la Salud (OMS), durante la 55 Asamblea Mundial de la Salud, abordó el tema y así comenzó a desarrollar distintas iniciativas y estrategias para mejorar las deficiencias en la atención de la salud. La Organización Panamericana de la Salud (OPS), que funciona como una oficina regional de la OMS, se sumó a este proyecto. De esta manera, se inició un movimiento mundial destinado al estudio y a la implementación de una política institucional de calidad y seguridad del paciente que no reconoció fronteras (Rodríguez-Herrera, 2017b).

En el 2005, la OMS creó "la Alianza Mundial para la seguridad del paciente" y lanzó el Primer Reto Global de Atención Segura OPS-OMS titulado: "Atención Limpia". La Caja Costarricense del Seguro Social (CCSS) designó al "Programa Institucional de Calidad y Seguridad del Paciente" como encargado de la gestión y monitoreo de lo relativo a estos retos globales OPS-OMS, en cumplimiento del compromiso gubernamental que firmó Costa Rica con la OPS respecto a la seguridad de los pacientes. Luego, vino el Segundo Reto Global: "Cirugía segura"; y actualmente se está trabajando en el Tercer Reto Global: "Medicación sin daños", lo que demuestra la aceptación internacional y la necesidad de este proyecto.

Así para la OMS, la seguridad del paciente se constituyó en un objetivo global y se impuso como un imperativo prioritario su promoción y aseguramiento para los gobiernos, sistemas de salud, universidades y asociaciones de profesionales de ciencias de la salud. 


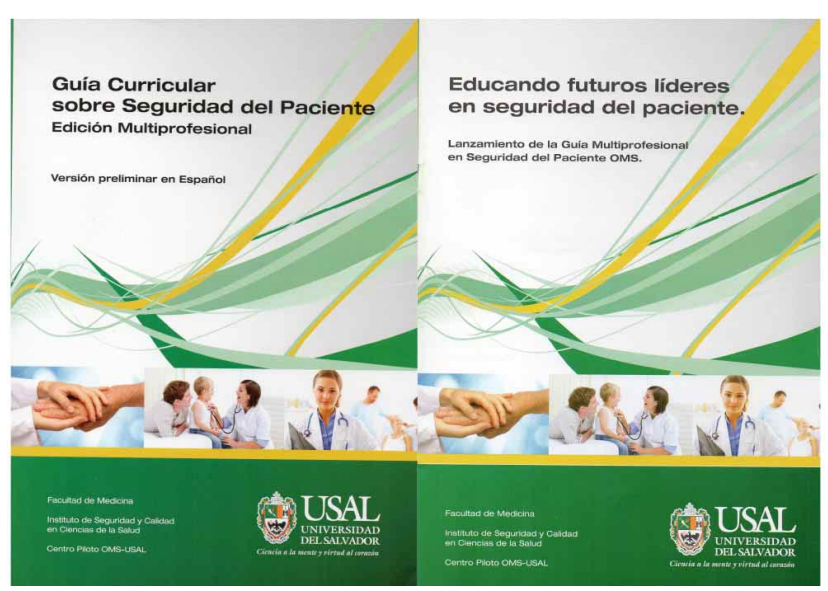

Fig. 9. Guía curricular multiprofesional sobre seguridad del paciente (OMS), traducida al castellano por el equipo de la Universidad del Salvador (USAL) y texto complementario del Instituto de Seguridad y Calidad en Ciencias de la Salud de dicha Universidad.

De esta manera, se promovió la enseñanza de la seguridad del paciente en las Escuelas de Medicina, a través de una guía curricular y se inició una nueva línea pedagógica con un programa especial.

En el 2013, la OMS designó a la Universidad del Salvador (USAL) de Argentina, centro piloto en Latinoamérica para el estudio de la evaluación de la implementación de las guías curriculares multiprofesionales para la enseñanza de la seguridad del paciente. A través de ellas, sugiere recomendaciones para lograr una atención segura y de alta calidad, involucrando a todos los profesionales de la salud (OMS, 2014; Martínez et al., 2010; Martínez, 2014) (Fig. 9).

\section{ANÁLISIS DEL PROBLEMA Y PROPUESTA DE SO- LUCIÓN}

Comprender la estructura del cuerpo es primordial para entender las funciones de los tejidos, los órganos y el cuerpo en general, a través de las ciencias básicas: estructurales y funcionales. La anatomía, la histología y la embriología, son los cimientos de la "casa" que uno construye. Una falla de éstos la hará vulnerable y la repercusión se notará en la atención del paciente durante el futuro ejercicio profesional.

Así como el ingeniero apela en su diario quehacer a los cálculos matemáticos básicos sin considerarlos como un fin sino como una herramienta; del mismo modo el médico, emplea cotidianamente los conocimientos anatómicos para una correcta interpretación de los problemas de salud de los pacientes. No se puede concebir la medicina si no se tiene un sólido conocimiento anatómico.
Además, la anatomía a través del material cadavérico -que facilita su enseñanza- brinda al alumno una primera aproximación a los valores humanísticos, al respeto y a la ética profesional.

No existe fórmula mágica para aprender "bien" anatomía en todos sus aspectos. Pero, sin duda, se debe aprender con sentido y significado. Para ello, la única forma es dedicarle el tiempo y la atención suficiente; y procurar relacionar los conocimientos adquiridos con nuestro propio cuerpo y el ajeno.

Por otra parte, a la inexperiencia de los alumnos en los estudios universitarios, que vienen de un aprendizaje escolar muy heterogéneo, debe sumarse el estrés que genera en ellos el aprendizaje de la anatomía por la cantidad de nuevos términos y temas que comprende. Además, los estudiantes vienen acostumbrados a las redes sociales y a la información anatómica disponible en internet, donde lo que frecuentemente predomina es la inmediatez, la brevedad y la superficialidad de los contenidos que se trasmiten.

Consideramos que el "constructivismo", es una teoría que puede guiar con menos dificultades el aprendizaje de la Anatomía. El estudiante es el centro del proceso; al reconocer, manipular e interpretar una pieza anatómica, está "haciendo", experiencia insustituible para aprender y no sólo escuchar y ver cómo ocurre, en las clases magistrales o cuando utiliza medios informáticos. De esta manera, suma: memoria, comprensión y análisis.

Por lo tanto, creemos que:

a) es primordial informar a los estudiantes sobre los beneficios y la importancia de un aprendizaje consciente de la anatomía para el futuro ejercicio profesional,

b) es fundamental el contacto con las piezas anatómicas en el museo y en el laboratorio o anfiteatro de disección,

c) resulta conveniente desarrollar la interrelación entre las ramas o subdivisiones de la Anatomía y fomentar el intercambio docente entre los que enseñan cada una de ellas. La anatomía facilita mucho la integración y la correlación de los conocimientos. La meta: aprendizaje anatómico con aplicación clínica: anatomía útil,

d) la Carrera de Medicina deberá dedicarle a "Anatomía" el tiempo necesario para poder realizar una formación más completa e integral de los futuros profesionales.

Este esquema, con estas cuatro propuestas, puede ser la base de modificaciones de la planificación curricular; y pueden ayudar a lograr una mayor seguridad de la práctica médica. El buen conocimiento anatómico disminuye los riesgos y evita errores en la atención.

La excelencia profesional y el buen desempeño para la seguridad de los pacientes, no se logra tomando atajos. Pen- 


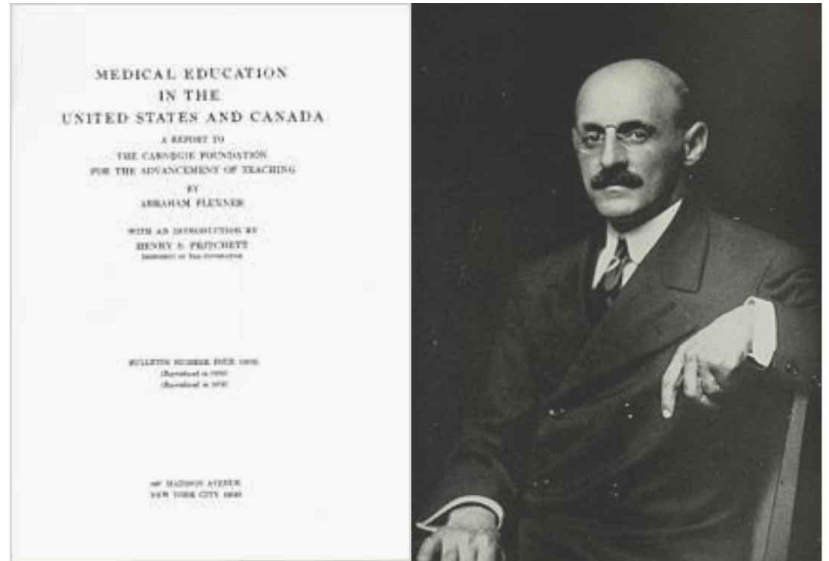

Fig. 10. Informe realizado por Abraham Flexner (1866-1959).

samos que la reducción del tiempo utilizado para el estudio de la anatomía en los programas curriculares resulta perjudicial para la formación médica; e indirectamente para quienes está destinada: los pacientes.

\section{DISCUSIÓN}

Por un lado, se reconoce que la anatomía tiene un valioso contenido para el médico, ya que tiene una gran cantidad de información que nos familiariza con los detalles más diminutos del maravilloso organismo humano.

La anatomía es una "ciencia básica estructural” del más alto valor, pues ella es la base de todas las otras disciplinas médicas, las cuales no podrían subsistir sin ella. Y esto se acrecienta cuando ella ofrece nociones preciosas para la práctica médico-quirúrgica, teniendo así el privilegio de ser útil para la humanidad (Bertelli, 1932).

Por otro lado, Flexner hace un siglo señaló las falencias de la enseñanza universitaria en la Carrera de Medicina. El objetivo de su estudio era cómo tener médicos con la mejor preparación para cubrir las necesidades de la población. El sostenía que a la sociedad se le debe asegurar que quien practica la medicina debía ser capaz y competente, tener los conocimientos, las habilidades y la actitud necesaria para ejercer como médico (Flexner, 1910) (Fig. 10).

Para ello, las Escuelas de Medicina deben estar bien equipadas y bien organizadas. La educación médica, desde entonces, se ha desarrollado y ha avanzado; y esto debe trasladarse a la enseñanza. Claro que esto tiene un costo. En estos casos la curva de aprendizaje de los diferentes procedimientos y técnicas, en los graduados bien formados, será más corta y segura para la atención de los pacientes. Por ejemplo, el novedoso uso de simuladores -que es la ficción- debe ser complementado con el uso de piezas anatómicas -que es la realidad- donde se observan las variedades anatómicas que demuestran la verdadera constitución del cuerpo humano. Esto prepara al futuro profesional para resolver situaciones en diversos escenarios, desde lo previsto hasta lo imprevisto. Por ello, son necesarios los museos y laboratorios para la enseñanza de la anatomía. De lo contrario la calidad de la misma se deteriora y se hace ineficiente.

Sin embargo, la meta no es hacer "técnicos médicos" sino "médicos humanísticos". También es posible que en el lugar donde se desempeñen, de manera transitoria, no dispongan de equipos sofisticados que lo ayuden a tomar decisiones.

Lamentablemente debemos reconocer que parte de nuestra realidad actual latinoamericana es compatible con la descripción flexneriana de hace más de cien años. Ello nos debería hacer reflexionar sobre este viejo problema aún presente. La "excelencia académica", término tan vapuleado hoy en el ámbito comercial educativo, resulta por ahora una utopía.

Si bien es cierto que la medicina ha cambiado gracias a los avances científicos y tecnológicos, también el mundo ha variado por diversas situaciones: a) envejecimiento de la población, deterioro del medio ambiente, nuevas enfermedades; b) accesibilidad y velocidad en las comunicaciones y la información; c) crisis de valores sociales; etcétera. Esto hace que el "modelo educativo médico tradicional" resulte insuficiente para las Facultades y Escuelas posflexnerianas (Bridge, 1965).

Sea cual fuere el modelo educativo, el estudiante debe aprender la materia de anatomía con el profesor, sin el profesor, a pesar del profesor y hasta en contra del profesor, si fuera necesario (Di Dio, 1998).

Como decíamos, la medicina y el mundo evolucionan; y nuevos paradigmas hacen cambiar viejos conceptos. A medida que la "tecnología" avanza y nos presenta nuevas herramientas de trabajo, los investigadores descubrirán nuevos universos en el cuerpo humano y así la "ciencia" también progresará (Di Dio). En definitiva, una suma de: 1) progreso científico; 2) avances tecnológicos; 3 ) explosión de las comunicaciones; 4) investigaciones educacionales; 5) exigencias de la sociedad; 6) presión económica; etcétera. En esta misma línea de pensamiento, los individuos cada vez más educados, pasaron a considerar la "protección de la salud" más como un derecho y menos como un privilegio económico, constituyendo un nuevo factor a considerar en la educación de los médicos.

Queda claro que en el campo de la educación médica hay muchas cosas que persisten: los objetivos son semejantes, los actores (alumnos y docentes) son los mismos, mu- 
chos de los medios para organizar, administrar y facilitar el aprendizaje tienen absoluta vigencia: planes de estudio, laboratorios, campos clínicos y bibliotecas.

Sin embargo, donde probablemente debamos avanzar más, es en las estrategias educacionales y en tener una mayor flexibilidad curricular.

En cuanto a las "estrategias", se deberá probar y evaluar el trabajo de casos, la búsqueda dirigida, los seminarios de integración, el trabajo de campo, la asignación de familias y comunidades para trabajar con ellas, las estancias en laboratorios y la participación en proyectos de investigación, entre otras.

En cuanto a la "flexibilidad curricular", se debe ver en varias dimensiones. Una de ellas tiene que ver con la formación universitaria del estudiante de medicina, que a diferencia de la mayoría de los otros estudiantes, sólo está en contacto directo con la Escuela o Facultad los primeros dos o tres años, para tener después muy poca concurrencia a la institución universitaria. Hablaría en este sentido de una "flexibilidad vertical" (Narro-Robles, 2004).

El estudio de Flexner sigue siendo importante. Sus conceptos y recomendaciones tienen vigencia y son aplicables en nuestro medio latinoamericano. Las disciplinas básicas con sus laboratorios, gabinetes y museos, siguen teniendo un papel fundamental en la formación del médico. El hospital y la enseñanza clínica también son claves en la tarea. Además, resultan indispensables los recursos complementarios: bibliotecas y ayudas educacionales. La educación médica de calidad tiene un costo; hay que asumirlo y encontrar el financiamiento requerido (Flexner, 1910).

Finalmente, las sociedades científicas que reúnen a los anatomistas muestran su interés participativo en cambiar esta situación, como la Asociación Panamericana de Anatomía (APA), creada en 1966 en México y miembro de la Federación Internacional de Asociaciones de Anatomistas (International Federation of Associations of Anatomists IFAA). La APA mantiene una intensa actividad desde su creación y tiene entre sus objetivos: "encauzar los sistemas de enseñanza de acuerdo con la evolución de las ciencias morfológicas" y "que la enseñanza... esté a cargo de profesionales adecuadamente preparados... y siga planes y programas actualizados" (Losardo, 2009). De esta manera, está habilitada a funcionar como "órgano de consulta" en toda América, ya que demuestra a través de su larga trayectoria, su compromiso con la docencia y la investigación de la anatomía en el continente. Su extensión académica es la Academia Panamericana de Anatomía, creada en el 2010 en Costa Rica.
La enseñanza de la anatomía es un factor indispensable en la preparación del médico. Un buen conocimiento de la anatomía integral ayuda -desde el inicio de su formación- a consolidar su madurez y a reforzar sus aspectos humanísticos, tan valorados por los pacientes hoy día y que contribuyen a la calidad de la atención médica.

Si bien el currículum médico está continuamente en un proceso de cambio y adecuándose a los nuevos avances científicos, el real conocimiento de la seguridad del paciente es diferente a las otras formas del conocimiento, en el sentido que éste debe ser aplicado en todas las diferentes áreas de la atención médica. Así, la seguridad del paciente aparece como una nueva disciplina o materia. El momento para difundir su conocimiento y su proyección es al comienzo, desde el primer año en la Escuela de Medicina (Martínez et al.).

\section{CONCLUSIONES}

La educación médica se basa en tres pilares: el estudiante, el docente y el programa curricular. Su objetivo es preparar a un individuo para brindar un servicio: la atención médica. Y tiene como fin a un usuario: el paciente. Y como principios o máximas: "Primero no dañar" (Primum non nocere), y "hacer el bien" (Bonum facere). La primera expresión es atribuida por algunos a Hipócrates; y la segunda está citada en el Juramento Hipocrático, con lo que se advierte que estos conceptos prácticamente nacieron con el "arte de curar".

La Anatomía permite entender la organización básica del cuerpo humano y los principios de funcionamiento de sus estructuras. En síntesis: estructura y función en equilibrio y armonía.

La falta o déficit de conocimiento anatómico dificulta, por ejemplo:

a) la buena exploración física del paciente;

b) la adecuada interpretación de la historia del motivo de la consulta, signos y síntomas en los cuadros clínicos;

c) la correcta interpretación de estudios o exámenes complementarios utilizados en la práctica médica;

d) la adecuada terminología de descripción de hallazgos patológicos, masas o tumoraciones, heridas, en relación o posición, en sus notas clínicas;

e) el correcto abordaje con técnicas mini-invasivas de las distintas regiones del cuerpo con fines diagnósticos o terapéuticos;

f) la prevención de errores de interpretación o comunicación entre colegas; 
El estudio incompleto y/o deficiente de la anatomía en la curricula genera un riesgo potencial de producir daño al paciente, que se puede manifestar a través de errores cometidos por el mismo médico.

En los últimos años, se han realizado esfuerzos para mejorar la seguridad de los pacientes. Sin embargo, en estos estudios sobre seguridad publicados, no se aborda la temática "anatómica" como factor de prevención de riesgos, errores y daños.

Pretendemos aportar algunas sugerencias en este campo, para que se pueda mejorar la seguridad de los pacientes.

1. El currículo de la Carrera de Medicina está en continuo cambio y adecuación a los avances científicos. La propuesta de esta comunicación es promover el aumento de las horas-cátedra y la calidad en la enseñanza de la Anatomía en el programa curricular de las Carreras de Medicina, ya que los estudiantes de hoy son los profesionales de mañana.

2. Además, los conocimientos anatómicos se deben integrar e incorporar a la práctica clínica; y mejorar los vínculos existentes entre la etapa formativa universitaria y la práctica clínica profesional.

3. Por otro lado, creemos que el currículo debe ser flexible para que el alumno pueda explorar horizontalmente campos, disciplinas y temas de orden médico de su interés. En la era de la globalización se debe favorecer esta flexibilidad transversal, que implique además rotaciones o visitas a otras Escuelas de Medicina del país e inclusive de otros países.

4. También sugerimos que los Colegios o Asociaciones profesionales se sumen a este desafío y colaboren revisando los contenidos curriculares y el tiempo de formación en anatomía humana, con el fin de que sean óptimos para lograr un profesional con un buen desempeño asistencial.

En resumen, somos optimistas frente al porvenir de la educación médica. Hay retos y tareas que deben ser enfrentados en el corto plazo; y también otros a mediano y largo plazo, que permiten estimular la imaginación y la creatividad. Lo que no hay duda, es que -actualmente- la responsabilidad es mayor e imperativa y el compromiso social se incrementó y se hizo relevante.

Estamos convencidos que estos cambios podrían mejorar la enseñanza y el aprendizaje de esta ciencia básica; y disminuir algunos riesgos en la atención sanitaria.

\section{AGRADECIMIENTOS}

A los Profesores por sus aportes y contribuciones: Dres. Jamnyce Altamirano (Universidad Nacional Autónoma de Nicaragua, Managua-Nicaragua y ex Presidenta de la Asociación Centroamericana y del Caribe de Anatomía); Gustavo Armand-Ugon (Universidad de la República, Montevideo-Uruguay); Manuel Arteaga-Martínez (Universidad Nacional Autónoma de México, México DFMéxico; Presidente de la Asociación Panamericana de Anatomía); Nelson Arvelo-D'Freitas (Universidad Central de Venezuela, Caracas-Venezuela); Omar Campohermoso-Rodríguez (Universidad Mayor de San Andrés, La Paz-Bolivia); Oscar A. Castillo (Universidad de Panamá, Panamá); Jordy Disla-Basora (Universidad Autónoma de Santo Domingo-República Dominicana); Héctor Guzmán-Duchén (Universidad Mayor de San Simón, Cochabamba-Bolivia; Presidente de la Asociación Boliviana de Anatomía); Blas Antonio Medina-Ruiz (Universidad Nacional de Asunción, Asunción-Paraguay); Jorge Moscol-Gonzales (Universidad Nacional de Villarreal, Lima-Perú); Antonio Soto-Paulino (Universidad Nacional Autónoma de México, México DF-México; Presidente de la Sociedad Mexicana de Anatomía); Nadir Eunice Valverde Barbato de Prates (Universidade de São Paulo, São Pablo-Brasil; Secretaria General de la Academia Panamericana de Anatomía).

\section{DEDICATORIA}

Los autores y colaboradores de este artículo quieren rendir homenaje al profesor doctor Rolando Cruz Gutiérrez (1934-2018), quien fomentaba la unión de los anatomistas de este continente. Mentor y fundador de los Simposios Ibero Latinoamericanos de Terminología y de la Academia Panamericana de Anatomía.

RODRÍGUEZ-HERRERA，R.; LOSARDO, R. J. \& BINVIGNAT, O. Human anatomy an essential discipline for patient safety. Int. J. Morphol., 37(1) :241-250, 2019.

SUMMARY: Excellent knowledge of human anatomy is critical for medical practitioners and clinicians in order to provide better healthcare and safety for their patients.

KEY WORDS: Human Anatomy; Patient's Safety; Medical Errors; Medical Education. 


\section{REFERENCIAS BIBLIOGRÁFICAS}

Arteaga-Martínez, M. \& García-Peláez, I. Embriología Humana y Biología del Desarrollo. $2^{\mathrm{a}}$ ed. Ciudad de México, Médica Panamericana, 2017.

Bertelli, D. Trattato di Anatomia Umana. $2^{\mathrm{a}}$ ed. Milán, Casa Editrice Dottor Francesco Vallardi, 1932.

Bogado-Yinde, L. A.; Ojeda-Fiore, H. A.; Medina-Ruiz, B. A. Estrategias Didácticas en la Enseñanza de la Anatomía Descriptiva y Topográfica: Una Mirada desde la Perspectiva de los Estudiantes, Egresados y Docentes de la FCM-UNA. Tesis de Magíster en Docencia Médica Superior. Asunción, Universidad Nacional de Asunción, 2015.

Bridge, E. M. Pedagogía Médica. Washington, Organización Panamericana de la Salud, 1965. Disponible en: http://www.who.int/iris/handle/ $10665 / 169992$

Di Dio, L. Tratado de Anatomía Aplicada. San Pablo, Polus, 1998.

Farache, S.; Losardo, R.; Perriard, D. \& Cambiasso, C. Opinión de los Estudiantes de Anatomía acerca de su Experiencia en la Asignatura. Rosario, Actas del XXVII Congreso de la Asociación Rioplatense de Anatomía, 1990a. pp.224.

Farache, S.; Perriard, D.; Losardo, R., Cambiasso, C. El Ciclo Básico Común $(C B C)$ como Sistema Introductorio de la Carrera de Medicina. Encuesta de 500 Alumnos. Rosario, Actas del XXVII Congreso de la Asociación Rioplatense de Anatomía. 1990b. pp.223.

Fernelii, I. Ambiani Universa Medicina. $6^{\mathrm{a}}$ ed. Hanoviae, Impenfis Claudii Marnii heredum, 1610.

Flexner, A. Medical Education in the United States and Canada. A Report to The Carnegie Foundation for the Advancement of Teaching. Nueva York, Bulletin Number Four, 1910.

Gardner, E.; Gray, D. J. \& O’Rahilly, R. Anatomía. Barcelona, Salvat. 1967.

Losardo, R. J. Pan-American Association of Anatomy: Historic review and eminent rules. Int. J. Morphol., 27(4):1345-52; 2009.

Losardo, R. J.; Binvignat Gutiérrez, O.; Cruz Gutiérrez, R. \& Aja Guardiola, $\mathrm{S}$. La anatomía y las prácticas adivinatorias en las antiguas civilizaciones. Rev. Asoc. Méd. Argent., 129(2):13-22, 2016.

Losardo, R. J.; Murcia, D. M.; Lacera Tamaris, V. \& Hurtado de Mendoza, W. Canon de las proporciones humanas y el hombre de Vitruvio. Rev. Asoc. Méd. Argent., 128(1):17-22, 2015a

Losardo, R. J.; Valverde Barbato de Prates, N. E.; Arteaga-Martínez, M.; Haltí Cabral, R. \& García-Peláez, M. I. International Morphological Terminology: More than anatomy, histology and embryology. Int. J. Morphol, 33(1):400-7, 2015b.

Mandarim-de-Lacerda, C. A. Breve História da Anatomia (com ênfase na Anatomia Cardiovascular). Río de Janeiro, Ediciones de la Universidade do Estado do Rio de Janeiro, 2010.

Martínez, J. C. Educando Futuros Líderes en Seguridad del Paciente. Lanzamiento de la Guía multiprofesional en Seguridad del Paciente OMS. Buenos Aires, Ediciones USAL, 2014.

Martínez, J. C.; Albanese, E.; Montarce, O.; Albanese, A. \& Cerviño, O. Proyecto pedagógico. La enseñanza de la seguridad y calidad en la asistencia médica y disciplinas aliadas en la Escuela de Medicina. Prensa Med. Argent., 97(1):52-5, 2010.

Montemayor Flores, B. G.; Herrera Vázquez, I. \& Soto Paulino, A. Analysis of the use of the Anatomical Terminology Between Students of Anatomy Courses at the Faculty of Medicine of the Universidad Nacional Autónoma de México. Int. J. Morphol., 34(4):1280-4, 2016.

Moscol-Gonzales, J. El conocimiento anatómico en el Perú preincaico. Rev. Asoc. Méd. Argent., 131(4):4-11, 2018.

Narro-Robles, J. La herencia de Flexner. Las ciencias básicas, el hospital, el laboratorio, la comunidad. Gac. Med. Mex., 140(1):52-5, 2004.

Organización Mundial de la Salud (OMS). Guía Curricular sobre Seguridad del Paciente. Edición Multiprofesional. Versión Preliminar en Español. Buenos Aires, Ediciones Universidad del Salvador (USAL), 2014.
Perriard, D. \& Losardo, R. Formación del personal docente auxiliar en Anatomía. Rev. Chil. Anat., 14(1):9-12, 1996.

Perriard, D. \& Losardo, R. La anatomía en una propuesta curricular integrada. Rev. Chil. Anat., 19(1):57-60, 2001.

Rodríguez-Herrera, R. Humanismo y medicina. Bol. CONAMED-OPS, 2(11):28-30, 2017a.

Rodríguez-Herrera, R. La seguridad del paciente y la ciber seguridad. Bol. CONAMED-OPS, 2(15):22-3, $2017 \mathrm{~b}$.

\author{
Dirección para correspondencia: \\ Dr. Ricardo Jorge Losardo \\ Lavalle 1844 - 50 "30" \\ Ciudad de Buenos Aires \\ ARGENTINA
}

\section{Email: ricardo.losardo@usal.edu.ar}

Recibido : 12-08-2018

Aceptado: 20-12-2018 